\title{
Analysis of the Quantitative and Mode of Traditional Ethnic in Consideration of Multi Characteristics
}

\author{
Li Moutao ${ }^{1}$ \\ ${ }^{1}$ College of Physical Education, Huangshan College, Huangshan Anhui, 245041 \\ ${ }^{1}$ limoutao1987@163.com
}

\begin{abstract}
Human sport action reconstruction is the current hot issue of image processing, against the feature of the problem, a new human action reconstruction method base on the combination of LLE and KRR is proposed. First, extract the human action framework against the color and deep information of the image and establish action training set and then transfer the training set into the motion vector library, calculate the low dimensional manifold using LLE algorithm and conduct correlation analysis to the manifold data, and finally make the predicted low dimensional manifold convergence action point mapped irreversibly to the low dimensional Euclidean space. This is the first application of KRR algorithm to solve traditional dimensionality reduction mapping irreversibility, so as to make the action reconstruction realized. The results show that: the construction action obtained by the convection action point is ideal, as the action process of the different degrees of freedom; the proposed human sport action construction has very good results.
\end{abstract}

Keywords: Local linear embedding; Sport action, Human action reconstruction; Nuclear ridge regression; Manifolds; Inverse mapping

\section{Introduction}

Human sport action is the topical issue in the field of computer vision and image processing. In recent year, with the rapid development of the image processing technology, the human action reconstruction has also been paid a great deal of attention of experts and scholars.

Currently, the study of the human action reconstruction is in its infancy at home at abroad. The video image processing [1-2], will often result in the immediate process image missing of the two action photos, which brings more difficult to the analysis of human action. The recognition of human action is the basis for reconstruction, the representing methods are: Histograms of Oriented Gradients (HOG) proposed by Dalai N[3], Hough Forests for Object Detection proposed by Gall[4], Locally Linear Embedding(LLE) proposed by Roweis S[9], and the human face recognition method based on LLE and SVM raised by Guofeng. Nilsson[15] and others research the high-dimensional data and propose the manifold use nuclear method for dimension reduction analysis. Lin[5] and others raised the method of linear interpolation image establishment combined with the existing image recognition method. And Liu Lijun[6] and others discuss the application of the interpolation method in the image. Interpolation is the traditional method for human action reconstruction. The coordinates of the human body sampling points are linearly inserted mainly through the recognition of human body action process, so as to achieve the reconstruction. But the body action of the reconstruction ignores the randomness and non-linearity of the human body action, causing the reconstruction inaccurate, stiff, uneven edges, even illogical, which is a simple linear interpolation process method. 
Given the Locally Linear Embedding(LLE) algorithm [7-14] can reduce the dimension of high-dimensional data. Kernel Ridge Regression (KRR) algorithm [1520] can effectively solve the problem of non-linear mapping. The author proposes a human body reconstruction method based on LLE and KRR. This method does not seek law intuitively from the human action, but conducts the process of mapping and inverse mapping through LLE dimensionality reduction method and algorithm, to solve the traditional dimensionality reduction without mapping, and then obtain the reconstructed human action, so as to effectively improve the disadvantage of the traditional algorithms. In this paper, the feasibility of the proposed method is analyzed through a large number of simulation experiments.

\section{LLE Algorithm}

LLE algorithm is a new method of dimensionality reduction for nonlinear data and the low dimensional data and the processed low dimensional data are able to be maintained the existing topology, with simple arithmetic operation, is the local optimization algorithm. LLE dimensionality reduction process is shown in Figure 1, which can map the data into the two-dimensional space by LLE. It can be seen that the data after dimensionality reduction can be well maintained the neighborhood characteristics of the original data.

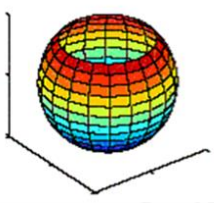

(a)Three-dimensional picture

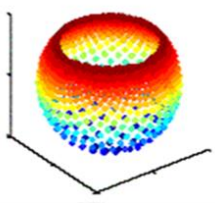

(b) Extracted sample points

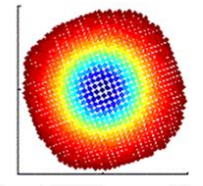

(c)Mapping to twodimensional space

Figure 1. LLE Dimensionality Reduction Figure

Assume there are $N$ input vector $X$, and output $Y$ can be obtained by LLE algorithm, which can be attributed to three aspects:

LLE calculation process is shown in Figure 2. the calculation steps are as follows:

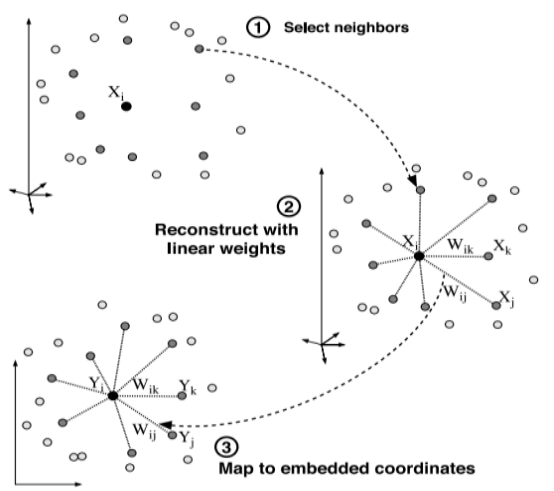

Figure 2. LLE Calculation Flowchart

Step 1: calculate $\mathrm{K}$ neighboring points of each sample point. Take the nearest $\mathrm{K}$ sample point with respect to the sample points as the $\mathrm{K}$ neighboring points of the sample point. 
Step 2: calculate the local reconstruction weight matrix of the sample point. Here let's define a cost error function:

$\min \varepsilon(W)=\sum_{i=1}^{N}\left|x_{i}-\sum_{j=1}^{k} w_{j}^{i} x_{i j}\right|^{2}$

Where, $x_{i j}(j=1,2, \cdots, k)$ are $\mathrm{k}$ neighboring points of $x_{i}, w_{j}^{i}$ is the weight between $x_{i}$ and $x_{i j}$, and meets $\sum_{j=1}^{k} w_{j}^{i}=1$ 。 To strike a $W$ matrix, a partial covariance matrix needs to be constructed:

$Q_{j m}^{i}=\left(x_{i}-x_{i j}\right)^{T}\left(x_{i}-x_{i j}\right)$

Combine the formula(2)and $\sum_{j=1}^{k} w_{j}^{i}=1$, and use the Lagrange multiplication, and then you can find the local optimal reconstruction weight matrix.

$$
w_{j}^{i}=\frac{\sum_{m=1}^{k}\left(Q^{i}\right)_{j m}^{-1}}{\sum_{p=1}^{k} \sum_{q=1}^{k}\left(Q^{i}\right)_{p q}^{-1}}
$$

In practice, $Q^{i}$ may be a singular matrix, when $Q^{i}$ must be regularized, namely:

$Q^{i}=Q^{i}+r I$

Where, $r$ is a regularization parameter and $I$ is a $k \times k$ unit matrix.

Step 3: Map all of the sample points to the low-dimensional space and the following criteria must be met:

$\min \varepsilon(Y)=\sum_{i=1}^{N}\left|y_{i}-\sum_{j=1}^{k} w_{j}{ }_{j} y_{i j}\right|^{2}$

Where, $\varepsilon(Y)$ is the loss function value, $y_{i}$ is the output vector of $x_{i}$, $y_{i j}(j=1,2, \cdots, k)$ are the $\mathrm{k}$ neighboring points of $y_{i}$, and meeting the following conditions:

$$
\sum_{i=1}^{N} y_{i}=0, \frac{1}{N} \sum_{i=1}^{N} y_{i} y_{i}^{T}=I
$$

Where, $I$ is $m \times m$ unit matrix, and here, $w_{j}^{i}(i=1,2, \cdots, N)$ can be stored in the sparse matrix $\mathrm{W}$ of $N \times N$. When $x_{j}$ is the neighboring point of $x_{i}, W_{i j}=w_{j}^{i}$, otherwise, $W_{i j}=0$, then the loss function can be rewritten as:

$\min \varepsilon(Y)=\sum_{i=1}^{N} \sum_{j=1}^{N} M_{i j} y_{i}^{T} y_{i}$

Where, $M$ is a symmetric matrix of $N \times N$, expressed as: 


$$
M=(I-W)^{T}(I-W)
$$

To obtain the minimum loss function value, the $\mathrm{Y}$ needs to be the feature vector of the minimum $\mathrm{M}$ non-zero feature values of $\mathrm{M}$. In the process, the feature values of $M$ should be in ascending order and the first feature value is almost close to zero, then it should be discarded. Usually take the feature vector corresponding to the feature value between $2 \sim m+1$ as the output.

\section{KRR Algorithm}

Kernel Ridge Regression(KRR) is an extension algorithm of the ridge regression. Ridge regression is a punishment form of the linear least-squares regression, and its minimized cost function is:

$$
L(W)=\frac{1}{n} \sum_{i=1}^{n}\left\|y_{i}-W^{T} x_{i}\right\|_{F}^{2}+\lambda\|W\|_{F}^{2}
$$

Where, $\lambda$ is a established regularization parameter. \|\|$_{F}$ is $\mathrm{F}$ norm, with $W \in R^{p \times d}$.

If you create a $X \in R^{n \times p}$ matrix, in which, $X$ each row is the output value from training input and associated with matrix $Y \in R^{n \times d}$, then the result can be written as:

$$
\arg \min L(W)=\left(X^{T} X+\lambda I_{p}\right)^{-1} X^{T} Y
$$

Where, $I_{p}$ is a $p \times p$ identity matrix, $f(x)=W^{T} x$ is the regression function and $x$ is the input vector.

This method can be extended by the use of nuclear transformation method, and thus it can deal with nonlinear problems, and then the regression function can be expressed as:

$$
f(x)=Y^{T}\left(K+\lambda I_{n}\right)^{-1} k(x)
$$

Where, $K \in R^{n \times n}$ is the Gram matrix of the training data. Gram matrix is formed by $K_{i j}=k\left(x_{i}, x_{j}\right)$ and $k(\cdot, \cdot) \quad$ is a Mercer kernel, $k(x)=\left(k\left(x, x_{1}\right), k\left(x, x_{2}\right), \cdots, k\left(x, x_{n}\right)\right)^{T}$ 。

We use the Gaussian kernel function (RBF):

$$
k_{\sigma}\left(x, x_{i}\right)=\exp \left(-\frac{\left\|x-x_{i}\right\|_{2}^{2}}{2 \sigma^{2}}\right)
$$

Where, $\sigma$ is an adjustment parameter, $\|.\|_{2}$ is Euclidean distance and $\sigma_{K}$ is the shape parameter, indicating the adjustment parameter $\sigma$ in KRR.

Select the value of $\sigma_{K}$ and put it in the formula (10) and (11), and then we can obtain the model able to be achieved.

\section{Realization of Human Sport Action Reconstruction}

The constitution of the human body has its representative and the human body should be characterized by many key points. Currently, the extraction of human skeleton is the recognized method of simplifying the complex body. The extraction of human body skeleton key points is shown in Figure 3. 


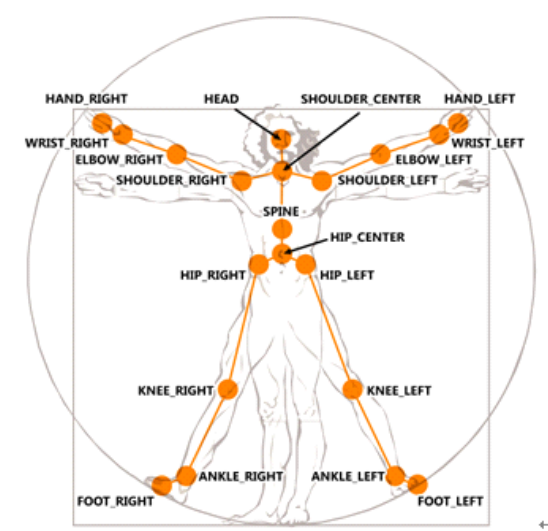

Figure 3. Human Body Skeleton Diagram

\subsection{LLE Mapping of Human Action}

We characterize the human body as the skeleton and in the proposed method, the low-dimensional manifold mapping can be divided into three steps:

(1) Supposing the human skeleton has $N$ points, and the two-dimensional coordinates of any pint is $\left(a_{i}, b_{i}\right)$, then the human action can be characterized as a high-dimensional vector $\left(a_{1}, b_{1}, a_{2}, b_{2}, \cdots, a_{N}, b_{N}\right)^{T}$.

(2) Collect a large number of human sport actions and make vector transfer of the process of every human body action, to form the action vector library.

(3) Conduct a dimensionality reduction to all action vectors according to LLE algorithm and obtain a low dimensionality manifold after dimensionality reduction.

Construct a $2 N$ dimensional Euclidean space. And any human action can be viewed as a point in Euclidean space. Suppose the number of the training action is $P$, then there are $P$ points in the Euclidean space. According to manifold leaning (manifold learning) theory, $\mathrm{P}$ points in Euclidean space constitute a high dimensional manifold, expressed as $S$.

According to the LLE dimensionality reduction theory in Section $1, m=2$ can be set, and $s$ is mapped to the two-dimensional manifolds, denotes as $s$. We consider human body action in $\mathrm{S}$ and after LLE notes the similar action after dimensionality reduction, it is still approximate. This mapping invariance ensures the neighboring points of the human body action in Euclidean space are still in the adjacent in S. The high dimensional manifolds neighboring points are shown in Figure 4.

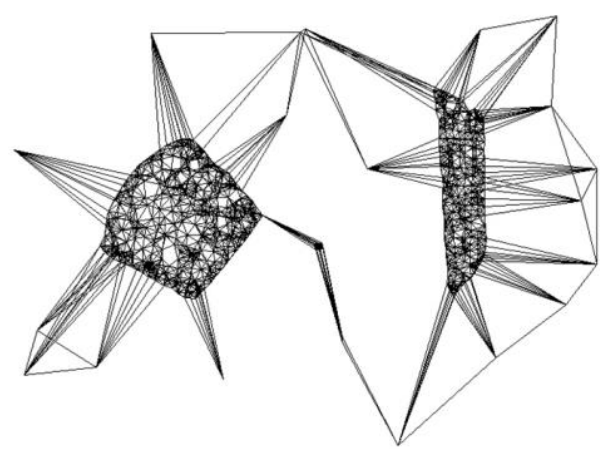

Figure 4. Neighboring Points Structure Chart 


\subsection{Reconstruction of Human Body Action}

The author believes that there is always a convergence relationship between any two human actions. Suppose $A$ and $B$ are the two human body actions, there are always $n$ convergence actions, making $A \rightarrow B$, as analyzed from the twodimensional flow after dimensionality reduction, as shown in Figure 5.

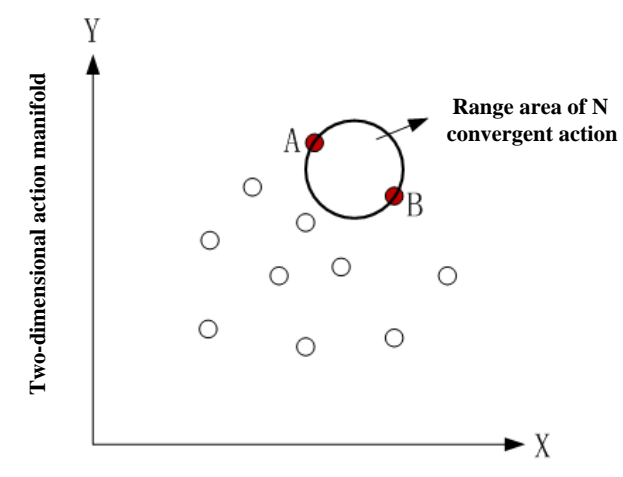

Figure 5. Analysis Chart of Two-dimensional Action Manifolds

As seen from Figure 5, the convergence action of A and B is always in a small area. The author believes it is in a circle with the approximate Euclidean distance between $\mathrm{A}$ and $\mathrm{B}$ as the diameter.

\section{Experimental Analysis}

In order to verify the effectiveness of the new method proposed in this paper, the author conducts programming in the Matlab 2012 platform, using the currently most advantageous video camera Kinect as the human body action training set. Given the shape parameter of KRR $\sigma_{K}$ and regularization parameter of $\lambda$, according to the different values of the different training sets, set $\sigma_{K}$ as 100, and $\lambda$ as 0.01 , using the training pictures of $512 \times 512$ pixel.

\subsection{Human Skeleton Extraction}

In the experiment, according to the human characteristic parts, select 20 key points for specific description, and we can extract the human skeleton combined the Kinect video capture function, as shown in Figure 6.

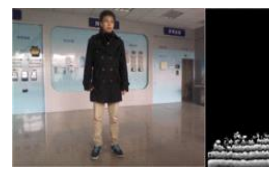

(a) Human Body Picture

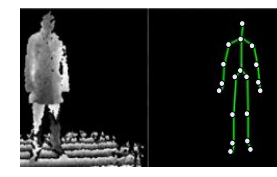

(b)Kinect Depth Picture

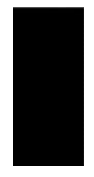

(c) Picture of Extracted Skeleton

Figure 6. Human Skeleton Extraction Picture

As can be seen form Figure 6(b), Kinect is better than the ordinary camera. The white points in (c) are the key points of the human body, and the human skeleton is 
depicted by the connection line between the two points. Take the pixels contained in the skeleton as the analysis objects.

\subsection{Human Body Action Two-Dimensional Manifold Generation Test}

For the 100 different actions, according to section 3.1, we set the adjacent points to be 8 and low dimensional manifold dimension to be 2 for mapping. The human body exercises sample is shown in Figure 7.
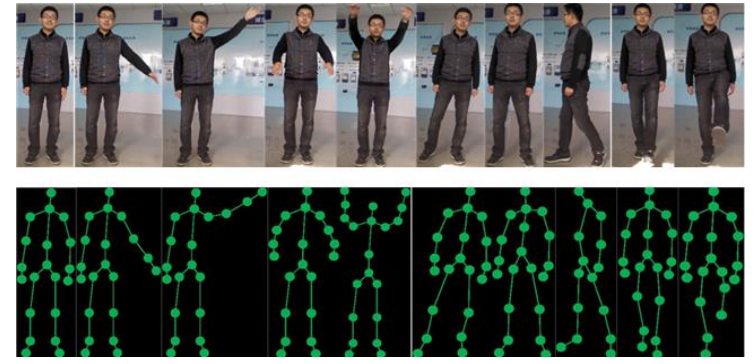

Figure 7. Human Body Exercises Sample Diagram

The two dimensional manifolds coordinates value after mapping do not have the physical sense, but there is space position law for each human body action from the high-dimensional mapping. And the manifolds after mapping are to be conducted with modeling, as shown in Figure 8.

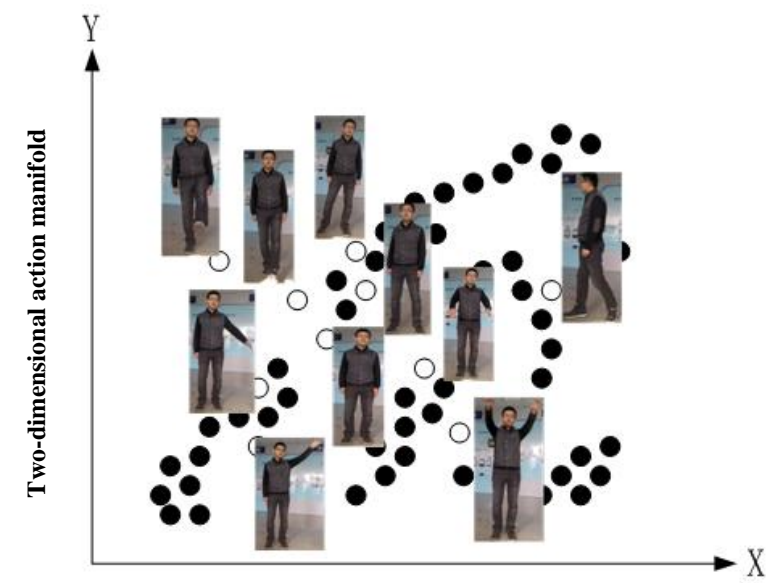

Figure 8. Human Body Two-Dimensional Manifold Chart

In Figure 8, the whiter points indicate the human body exercise action in Figure 7. It can be seen from Figure8: the action points of the two-dimensional manifolds after mapping have neighboring relations and the Euclidean distance between the similar actions is often very small.

\subsection{Reconstruction Test of Human Body action}

5.3.1. Selection of Convergence Action Point: The human body action involves multiple freedom degrees, against which, the human body action are reconstructed in the test. For the different training set, in the action convergence area, they contain different numbers of training actions. Supposing the convergence area of A and B actions contains $\mathrm{W}$ action points, we calculate the Euclidean distance between $\mathrm{W}$ 
action points and points A and B. According to the ascending order of the Euclidean distance between the action point to point $\mathrm{A}$, the $\mathrm{w}$ action points are recorded as $1 \sim w$, as shown in Figure 9.

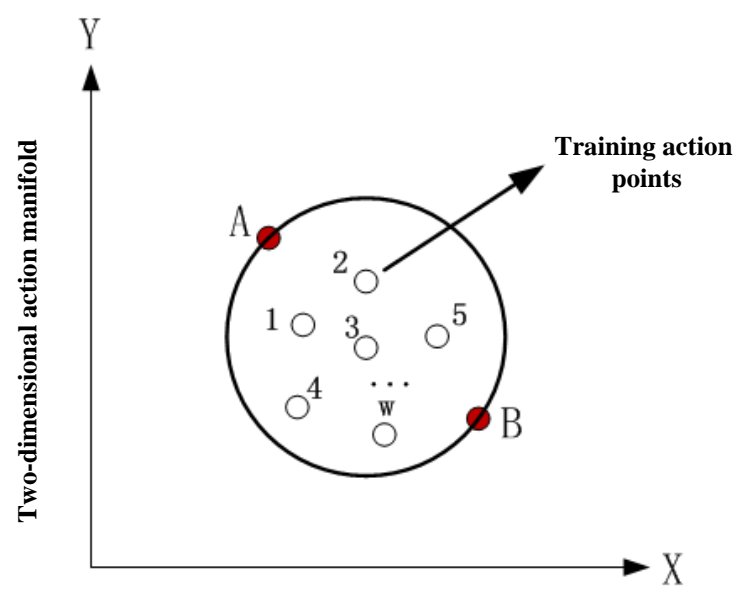

Figure 9. Training Actions Profile

According to the circular area characteristics, the smaller the Euclidean distance between the training point and point $\mathrm{A}$, the bigger the Euclidean distance between it and point B. We describe its characteristics, as shown in Figure 10.

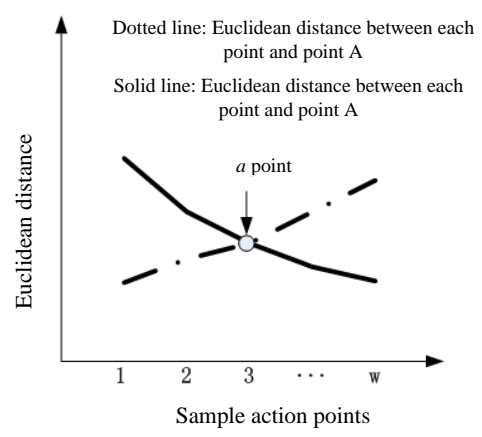

Figure 10. Euclidean Distance Graph

In Figure 10, $\alpha$ point is the intersection of the two curves. Set $\omega_{1}, \omega_{2}$ as the convergence point of $\mathrm{A}$ and $\mathrm{B}, \alpha$ coordinate is, A coordinate is $\left(x_{1}, y_{1}\right)$, and $\mathrm{B}$ coordinate is $\left(x_{2}, y_{2}\right)$. Then

$$
\omega_{1}=\tau_{1}\left(x_{1}, y_{1}\right)+\left(1-\tau_{1}\right)(x, y) \omega_{2}=\tau_{2}\left(x_{2}, y_{2}\right)+\left(1-\tau_{2}\right)(x, y)
$$

Where, the values of $0<\tau_{1}<1, \quad 0<\tau_{2}<1, \tau_{1} 、 \tau_{2}$ determine the outcome of reconstruction. We select the reconstruction with the most ideal effect as the result.

5.3.2. Reconstruction of Human Action with Smaller Freedom: We firstly conduct reconstruction for the human body action with smallest freedom, with the experimental action shown in Figure 11. 


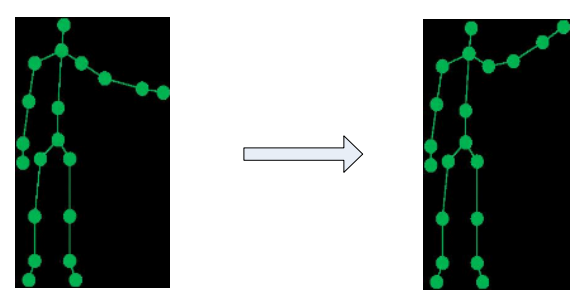

Figure 11. Small DOF Action Experiment Picture

Figure 11 shows the human left hand stretching process, with the actions defined as $\mathrm{A}$ and $\mathrm{B}$, according to (1) of this section and Section 4.2, the most ideal convergence action points are selected, for which the reconstruction is conducted and then conduct local correction process to the pixel using the color gradient change. The correction method is as following:

Calculate the gradient of the human body color pixel color in the reconstruction action figure and the adjacent pixels with large color variance are considered as deviation points. We conduct plan modeling (with the human critical points labeled and the skeleton established) to the reconstruction image after correction, with the color value of the deviation values replaced by the average value of the nondeviation color, and obtain the action model image as shown in Figure 12.
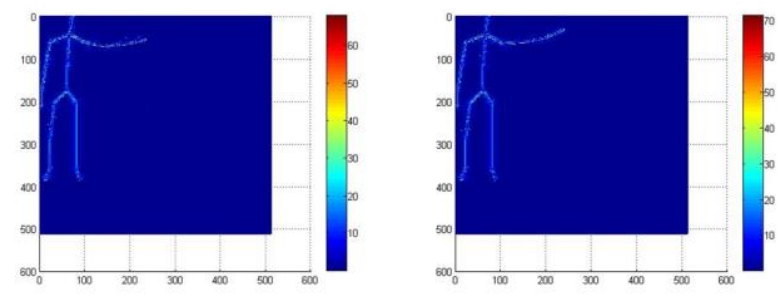

(a) Matlab Construction of Action Image

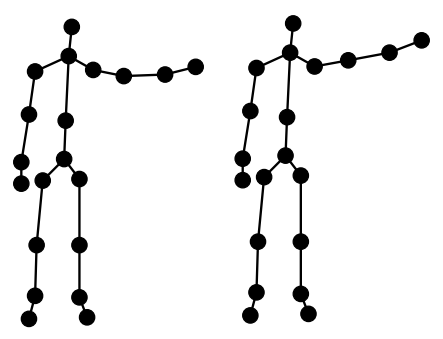

(b) Big DOF Action Experiment Picture

\section{Figure 12. Convergence Action Reconstruction Picture}

Figure 12 contains two reconstruction results, expressing the two reconstruction convergence action of left hand stretching respectively with line and dotted line. We can clearly see that the reconstructed human body convergence action is preferable, which is a logical reconstruction.

5.3.3. Reconstruction of Human Action with Bigger Freedom: In order to verify the reconstruction result human body action with the big freedom, we use the experiment figure of big freedom, as shown in Figure 13. 


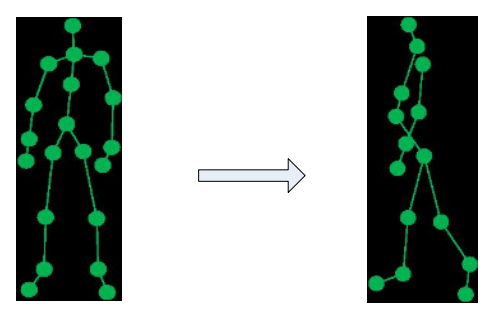

Figure 13. Big DOF Action Experiment Picture

Figure 13 shows the body turning action, with the same test method with (2) of this section, and the results obtained are shown in Figure 14.
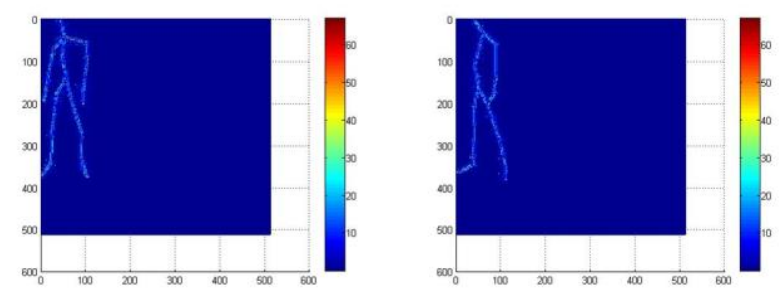

(a) Matlab Construction of Action Image

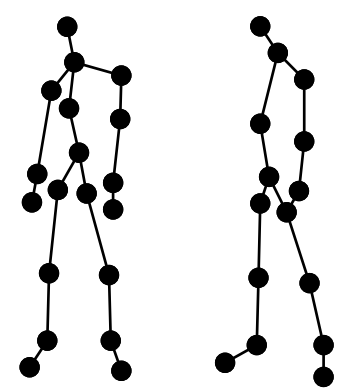

(b) Action Image Plane after Plane Modeling

Figure 14. Convergence Action Reconstruction Picture

It can be seen from Figure 14: the human body action under big freedom can better reconstruct the convergence action, which shows that the algorithm in this paper has strong ability to adapt to the action reconstruction.

\section{Conclusion}

This paper has deep study and analysis to the human body action and raises a new human action reconstruction method based on LLE and KRR algorithm, which firstly conducts LLE dimensionality reduction to the human body action library and obtain the convergence action point through analysis of the action point after dimensionality reduction, and then conduct non-linear mapping to the predicted convergence action point using KRR algorithm for the reconstruction of human body action. The results show: KRR algorithm can effectively solve the problem that cannot be mapped by LLE algorithm, which is the important basis for the recovery of high-dimensional manifold body action, having very important significances on the optimization of LE algorithm. The obtained reconstruction actions are very accurate and logical, thus effectively solve the problem that the image deletion cannot be recovered, providing an important theoretical basis for the application of the algorithm in this paper in other areas. But all of the human body reconstruction action figures still have local unsmooth area, so the future work is to 
optimize the operation of reconstruction and recover the action with the threedimensional tools, to obtain a more smooth, clear and beautiful reconstruction action.

\section{Acknowledgment}

The research is Supported by Key projects of the Foundation for Young Talents in Anhui Province (2013SQRW082ZD).

\section{References}

[1] K. Wang, "Overcoming Hadoop Scaling Limitations through Distributed Task Execution".

[2] S. Zhang, X. Zhang and X. Ou, "After we knew it: empirical study and modeling of cost-effectiveness of exploiting prevalent known vulnerabilities across iaas cloud", Proceedings of the 9th ACM symposium on Information, computer and communications security, ACM, (2014).

[3] W. Gu, Z. Lv and M. Hao, "Change detection method for remote sensing images based on an improved Markov random field", Multimedia Tools and Applications, (2016).

[4] Z. Lu, C. Esteve, J. Chirivella and P. Gagliardo, "A Game Based Assistive Tool for Rehabilitation of Dysphonic Patients", 3rd International Workshop on Virtual and Augmented Assistive Technology (VAAT) at IEEE Virtual Reality 2015 (VR2015), Arles, France, IEEE, (2015).

[5] Z. Chen, W. Huang and Z. Lv, "Uncorrelated Discriminant Sparse Preserving Projection Based Face Recognition Method", Multimedia Tools and Applications, (2016).

[6] Z. Lv, A. Halawani, S. Feng, H. Li and S. U. Rehman, "Multimodal Hand and Foot Gesture Interaction for Handheld Devices", ACM Transactions on Multimedia Computing, Communications, and Applications (TOMM), 11, 1s, Article 10, (2014) October, pp. 19.

[7] K. Leng, W. Shi, J. Chen and Z. Lv, "Designing of a I-shaped less-than-truckload cross-dock: A simulation experiments study", International Journal of Bifurcation and Chaos, (2015).

[8] Y. Lin, J. Yang, Z. Lv, W. Wei and H. Song, "A Self-Assessment Stereo Capture Model Applicable to the Internet of Things", Sensors, (2015).

[9] N. Lu, C. Lu, Z. Yang and Y. Geng, "Modeling Framework for Mining Lifecycle Management", Journal of Networks", vol. 9, no. 3, (2014) January, pp. 719-725.

[10] Y. Geng and K. Pahlavan, "On the accuracy of rf and image processing based hybrid localization for wireless capsule endoscopy", IEEE Wireless Communications and Networking Conference (WCNC), (2015) March

[11] G. Liu, Y. Geng and K. Pahlavan, "Effects of calibration RFID tags on performance of inertial navigation in indoor environment", 2015 International Conference on Computing, Networking and Communications (ICNC), (2015) February.

[12] J. He, Y. Geng, Y. Wan, S. Li and K. Pahlavan, "A cyber physical test-bed for virtualization of RF access environment for body sensor network", IEEE Sensor Journal, vol. 13, no. 10, (2013) October, pp. 3826-3836.

[13] W. Huang and Y. Geng, "Identification Method of Attack Path Based on Immune Intrusion Detection", Journal of Networks, vol. 9, no. 4, (2014) January, pp. 964-971.

[14] G. Bao, L. Mi, Y. Geng, M. Zhou and K. Pahlavan, "A video-based speed estimation technique for localizing the wireless capsule endoscope inside gastrointestinal tract", 2014 36th Annual International Conference of the IEEE Engineering in Medicine and Biology Society (EMBC), (2014) August.

[15] D. Zeng and Y. Geng, "Content distribution mechanism in mobile P2P network", Journal of Networks, vol. 9, no. 5, (2014) January, pp. 1229-1236.

[16] M. Zhou, G. Bao, Y. Geng, B. Alkandari and X. Li, "Polyp detection and radius measurement in small intestine using video capsule endoscopy", 2014 7th International Conference on Biomedical Engineering and Informatics (BMEI), (2014) October.

[17] G. Yan, Y. Lv, Q. Wang and Y. Geng, "Routing algorithm based on delay rate in wireless cognitive radio network", Journal of Networks, vol. 9, no. 4, (2014) January, pp. 948-955.

[18] G. Bao, L. Mi, Y. Geng and K. Pahlavan, "A computer vision based speed estimation technique for localiz ing the wireless capsule endoscope inside small intestine", 36th Annual International Conference of the IEEE Engineering in Medicine and Biology Society (EMBC), (2014) August.

[19] X. Song and Y. Geng, "Distributed community detection optimization algorithm for complex networks", Journal of Networks, vol. 9, no. 10, (2014) January, pp. 2758-2765.

[20] J. Hu and Z. Gao, "Distinction immune genes of hepatitis-induced heptatocellular carcinoma", Bioinformatics, vol. 28, no. 24, (2012), pp. 3191-3194.

[21] J. Hu, Z. Gao and W. Pan, "Multiangle Social Network Recommendation Algorithms and Similarity Network Evaluation”, Journal of Applied Mathematics, (2013). 
[22] J. Hu and Z. Gao, "Modules identification in gene positive networks of hepatocellular carcinoma using Pearson agglomerative method and Pearson cohesion coupling modularity", Journal of Applied Mathematics, (2012).

[23] X. Zhang, "Spike-based indirect training of a spiking neural network-controlled virtual insect", 2013 IEEE 52nd Annual Conference on Decision and Control (CDC). IEEE, (2013).

[24] W. Ou, Z. Lv and Z. Xie, "Spatially Regularized Latent topic Model for Simultaneous object discovery and segmentation", The 2015 IEEE International Conference on Systems, Man, and Cybernetics SMC, (2015).

[25] K. Wang, "Using Simulation to Explore Distributed Key-Value Stores for Exascale System Services", 2nd Greater Chicago Area System Research Workshop (GCASR), (2013).

[26] Y.Wang, Y. Su and G. Agrawal, "A Novel Approach for Approximate Aggregations Over Arrays", In Proceedings of the 27th international conference on scientific and statistical database management, ACM, (2015).

[27] Z. Lv, A. Halawani, S. Feng, S. u. Rehman and Ha. Li, “Touch-less Interactive Augmented Reality Game on Vision Based Wearable Device", Personal and Ubiquitous Computing, (2015).

\section{Author}

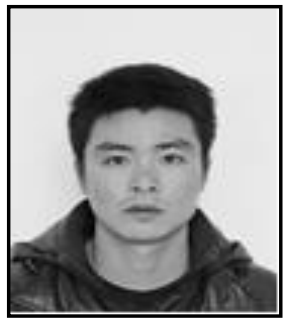

Li Moutao, received his M.S. degree in National Traditional Sports from Jishou University in Hunan Province, China. He is currently an assistant professor of Sports Science at Huangshan University. His research interest is mainly in the area of Sports Culture and National Traditional Sports. He has published several research papers in scholarly journals in the above research areas and has participated in several books. 